\title{
APROVEITAMENTO DE PRECURSORES LIGNOCELULÓSICOS PARA PRODUÇÃO DE ACETATO DE CELULOSE
}

\author{
Mayara da S. Santos ${ }^{\mathrm{a}}$, Heliane R. do Amaral ${ }^{\mathrm{a}}$, Daniel F. Cipriano ${ }^{\mathrm{a}}$, João V. T. Ferreti ${ }^{\mathrm{b}}$, Carla da S. Meireles ${ }^{\mathrm{b}}$, Jair C. C. \\ Freitas $^{\text {a e Reginaldo B. dos Santos }}{ }^{\mathrm{c}, * \text {,i) }}$

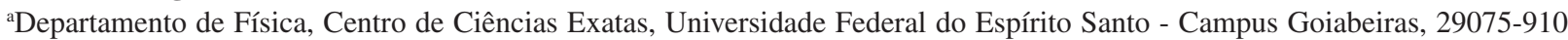 \\ Vitória - ES, Brasil \\ ${ }^{b}$ Departamento de Ciências Naturais, Centro Universitário Norte do Espírito Santo, Universidade Federal do Espírito Santo, \\ 29932-540 São Mateus - ES, Brasil \\ 'Departamento de Química, Centro de Ciências Exatas, Universidade Federal do Espírito Santo - Campus Goiabeiras, 29075-910 \\ Vitória - ES, Brasil
}

Recebido em 03/09/2019; aceito em 18/12/2019; publicado na web em 26/03/2020

\begin{abstract}
USING LIGNOCELLULOSIC PRECURSORS FOR THE PRODUCTION OF CELLULOSE ACETATE. The feasibility of obtaining cellulose acetate from lignocellulosic precursors is of great interest from the environmental, economic and technological points of view. Herein, sugarcane bagasse and vegetable sponge (Luffa cylindrica) were used as precursors to produce cellulose using the organosolv extraction procedure. From the extracted celluloses, the production of cellulose acetate was carried out by the homogeneous acetylation method. The obtained materials were characterized by FTIR, solid-state ${ }^{13} \mathrm{C}$ NMR, XRD, TG and DSC techniques. The results revealed the success of both the extraction and the acetylation processes, which provided cellulose with high crystallinity and cellulose acetates with high degrees of substitution (cellulose triacetates).
\end{abstract}

Keywords: lignocellulosic materials; agricultural waste; cellulose acetate; sugarcane bagasse; vegetable sponge; biorefinery.

\section{INTRODUÇÃO}

Os avanços na área de biotecnologia e a crescente difusão do conceito de biorrefinaria têm estimulado o uso integral da biomassa, com o objetivo de agregar valor às cadeias produtivas e diminuir os impactos ambientais. ${ }^{1-3}$ Para exemplificar essa tendência, a palha da cana de açúcar tem sido amplamente usada para obtenção de etanol; ${ }^{4}$ em outro exemplo, resíduos da agroindústria como bagaço de cana, fibra de coco e farelo de soja foram adicionados ao amido de mandioca na obtenção de compósitos na forma de embalagens biodegradáveis (bandejas) para uso na indústria alimentícia. ${ }^{5}$ Ribeiro, Carvalho e Geris $^{5}$ extraíram furfural (solvente orgânico amplamente usado nas indústrias químicas e farmacêuticas) a partir de sabugos de milho. A produção da enzima celulase a partir dos precursores bagaço e palha de cana, palha de soja e de arroz e sabugo de milho foi discutida por Castro e Pereira $\mathrm{Jr}^{5}{ }^{5} \mathrm{~A}$ biomassa lignocelulósica ainda pode ser usada para a obtenção de vários produtos de grande interesse tecnológico e econômico, incluindo carvões vegetais, carvões ativados, biopolímeros e biocombustíveis. ${ }^{6,7}$ Materiais celulósicos avançados também podem ser obtidos a partir de precursores lignocelulósicos (como bagaço de cana e bucha vegetal), dentre os quais podem ser mencionados nanocelulose, metilcelulose, acetato de celulose. ${ }^{8-14}$

Um derivado da celulose de grande importância comercial é o acetato de celulose (AC). Espera-se que até 2026 o mercado de AC movimente aproximadamente 7,96 bilhões de dólares. ${ }^{15,16} \mathrm{~A}$ aplicação desse produto em fibras, plásticos, revestimentos resistentes a solventes, produção de membranas, entre outros, tem sido amplamente investigada. ${ }^{17-20} \mathrm{O}$ AC é obtido através da reação de acetilação, na qual ocorre a esterificação dos grupos hidroxila presentes nos resíduos de glicose. Uma vez que cada resíduo de glicose possui três hidroxilas (cada uma ligada aos carbonos 2, 3 e 6), existe a possiblidade de se obterem materiais com diferentes graus de substituição (GS), isso é,

*e-mail: reginaldo.santos@ufes.br o número médio de hidroxilas esterificadas pode variar de zero, para a celulose, a três, para o triacetato de celulose (TAC). ${ }^{21}$

A obtenção de AC a partir de precursores lignocelulósicos (como bagaço de cana) para aplicações em materiais avançados tem sido abordada em diferentes estudos. Candido e colaboradores ${ }^{14}$ sintetizaram AC a partir de bagaço de cana com GS de 2,52 e aplicaram esse material na forma de membranas. Concluíram o trabalho demonstrando que as membranas obtidas a partir do AC, proveniente do bagaço de cana, podem ser aplicadas em processos de dessalinização, separação de gases e liberação controlada de drogas. Melo Brites e colaboradore $^{22}$ produziram nanofibras de triacetato de celulose a partir de bagaço de cana para imobilização da enzima bromelina. Os resultados mostraram que esse material possui uma elevada atividade celular. Além disso, testes in vitro foram realizados para avaliar semiquantitativamente a liberação controlada da enzima bromelina, que ocorreu completamente em três dias. Ramphul e colaboradores ${ }^{23}$ também extraíram celulose a partir do bagaço de cana, converteram-na em acetato de celulose e finalmente preparam uma blenda com o polímero ácido poliláctico ou polidioxanona. Esse material sofreu eletrofiação para obtenção de plataformas que foram usadas para o crescimento de células de fibroblastos de ratos. Estudos preliminares mostraram o potencial de biomineralização dessa plataforma pelo depósito de nanohidroxiapatita.

São descritas na literatura várias rotas para obtenção de AC a partir de materiais de biomassa, sendo a principal diferença entre elas a possibilidade de acetilação do precursor in natura em comparação com a acetilação da celulose obtida após extração. Chen e colaboradores $^{24}$ acetilaram o bagaço de cana com estér vinílico e líquido iônico para obtenção de bioplásticos; Das e colaboradores ${ }^{25}$ acetilaram a celulose proveniente da casca de arroz usando anidrido acético e iodo em uma rota ambientalmente amigável, isto é, livre de solventes. Abraham e colaboradores ${ }^{8}$ acetilaram nanocristais de celulose usando iodo e anidrido acético, obtendo um aerogel que exibiu propriedades multifuncionais de hidrofobicidade, oleofilicidade 
e lipofilicidade. Contudo, o método mais reportado na literatura e comercialmente empregado ${ }^{26}$ usa ácido acético/anidrido acético e ácido sulfúrico (como catalisador) para obtenção de $\mathrm{AC}$ a partir da celulose, sendo esse o método utilizado neste trabalho.

O Brasil é mundialmente reconhecido como um grande produtor agrícola; estimativas do Instituto Brasileiro de Geografia e Estatística (IBGE) mostraram que no ano de 2018 o Brasil produziu aproximadamente 2 bilhões de toneladas de produtos agrícolas (tais como: algodão, amendoim, arroz, banana, batata inglesa, cacau, feijão, fumo, laranja, mandioca, milho, soja, sorgo, tomate, trigo e uva). Por outro lado, a produção de cana de açúcar, no período de janeiro a setembro de 2019, foi de mais de 6 bilhões de toneladas. ${ }^{27}$ Esse cenário demonstra a importância de estudos que investiguem aplicações avançadas que possam agregar valor aos subprodutos oriundos dessa atividade agrícola (como o bagaço de cana) ${ }^{28}$ Por outro lado, a bucha vegetal é um material de biomassa subutilizado no Brasil, com uso principalmente associado à produção de itens de higiene pessoal, fabricação de palmilhas para sapatos, tapetes e chapéus $;^{29,30}$ esse precursor apresenta também potencial para obtenção de celulose e seus derivados, como acetato de celulose.

Nesse contexto, o objetivo deste trabalho foi demonstrar que a mesma metodologia pode ser empregada para diferentes tipos de precursores lignocelulósicos (bagaço de cana e bucha vegetal), visando a obtenção de acetato de celulose com alto grau de substituição. Os resultados obtidos contribuem para ampliar as possibilidades de aproveitamento de materiais lignocelulósicos abundantes no Brasil, sendo um deles (o bagaço de cana) um importante subproduto da agroindústria e o outro (bucha vegetal) um material ainda pouco investigado como precursor para obtenção de produtos celulósicos.

\section{PARTE EXPERIMENTAL}

\section{Materiais}

Todos os reagentes foram adquiridos de fornecedores locais: ácido nítrico (65\%, Neon); álcool etílico (70\%, Alphatec); ácido sulfúrico (98\%, Êxodo); hidróxido de sódio (99\%, Vetec); carbonato de sódio (99,5\%, Synth); ácido acético (99,8\%, Neon); anidrido acético (97\%, Dinâmica). Amostras de bagaço de cana foram fornecidas por produtores locais do município de Conceição da Barra no Estado do Espírito Santo. A bucha vegetal foi comprada em supermercados locais na cidade de Vitória no Estado do Espírito Santo. Todos os reagentes foram de grau analítico e utilizados sem purificação.

\section{Metodologia}

\section{Extração da celulose a partir do bagaço de cana (BC) e da bucha} vegetal $(B U)$

A extração de celulose foi realizada de acordo com o procedimento descrito por Amaral e colaboradore ${ }^{10}$ utilizando a metodologia de extração organossolve. Nesse processo, a celulose é extraída em três etapas. Na primeira etapa, a bucha vegetal (12 g), sem a casca externa, e o bagaço de cana $(30 \mathrm{~g}$ ) reagiram, sob refluxo em uma temperatura de $\sim 100{ }^{\circ} \mathrm{C}$, com $500 \mathrm{~mL}$ de uma solução etanólica de ácido nítrico $20 \%$ por 3 h. Após filtração, o material sólido reagiu com $\mathrm{NaOH}$
1,00 mol L $^{-1}$, sob agitação moderada, a temperatura ambiente, por $1 \mathrm{~h}$ - essa foi a segunda etapa. Depois de filtrado, o material sólido foi lavado com uma solução $20 \%$ de ácido acético por $5 \mathrm{~min}$, sob agitação magnética. Finalmente, o material foi novamente filtrado, lavado com água destilada e seco em estufa a $60^{\circ} \mathrm{C}$ por $12 \mathrm{~h}$.

$\mathrm{O}$ rendimento do processo foi determinado em termos da massa de celulose obtida com relação à massa inicial de precursor, enquanto que o rendimento da extração de celulose foi determinado relacionando o rendimento do processo com os teores de celulose presentes nos precursores, obtidos a partir da caracterização química realizada segundo os métodos descritos por Morais e colaboradores; ${ }^{31}$ os resultados dessa análise são apresentados no Material Suplementar, bem como os valores de rendimento do processo e da extração de celulose.

\section{Preparação do acetato de celulose (AC)}

A obtenção do AC foi conduzida pelo método de acetilação homogênea que também foi descrito por Amaral e colaboradores ${ }^{10}$ à temperatura ambiente. Na reação de acetilação 2,0 g de celulose, extraídas na etapa anterior, foram agitadas magneticamente com $70 \mathrm{~mL}$ de ácido acético glacial por $30 \mathrm{~min}$. Após esse tempo, foram adicionados $18 \mathrm{~mL}$ de uma solução de $0,02 \mathrm{~g} \mathrm{~mL}^{-1}$ de $\mathrm{H}_{2} \mathrm{SO}_{4} \mathrm{em}$ ácido acético glacial e a agitação magnética foi mantida por $25 \mathrm{~min}$ adicionais. $\mathrm{O}$ material foi filtrado sob vácuo e retornado ao frasco reacional. Ao filtrado foram adicionados $64 \mathrm{~mL}$ de anidrido acético e a mistura ficou sob agitação magnética por $30 \mathrm{~min}$. O sistema foi mantido em repouso por $24 \mathrm{~h}$. Em seguida, sob agitação constante, todo o material foi precipitado com $~ 300 \mathrm{~mL}$ de água destilada e filtrado a vácuo. $\mathrm{O}$ precipitado obtido foi neutralizado com uma solução $10 \%$ de $\mathrm{Na}_{2} \mathrm{CO}_{3}$ até $\mathrm{pH} \approx 7$, lavado com água destilada, filtrado e seco em estufa a $90{ }^{\circ} \mathrm{C}$ por $4 \mathrm{~h}$. O produto final foi pesado e o rendimento da extração foi calculado a partir da Equação 1, que relaciona a massa (em g) de acetato de celulose obtida $\left(m_{A C}\right)$ com a quantidade esperada a partir de 2,0 g de celulose:

$$
R_{\text {acetilasão }}=\frac{m_{A C}}{\left(\frac{2,0 \mathrm{~g}}{162 \mathrm{~g} \mathrm{~mol}^{-1}} \times M M_{A C}\right)} \times 100 \%
$$

em que $M M_{A C}$ é o valor da massa molar de um monômero de acetato de celulose considerando o grau de substituição do material acetilado.

A Tabela 1 apresenta a nomenclatura adotada e a descrição dos produtos celulósicos obtidos, tanto na extração da celulose quanto na preparação do acetato.

\section{Caracterizações}

\section{Determinação da Massa Molar Viscosimétrica $\left(M_{v}\right)$ e do Grau de Polimerização (GP) para a celulose}

Para determinação dos valores de GP e $M_{v}$ das amostras de celulose seguiu-se o procedimento descrito na norma ABNT NBR 7730. Para as medidas de viscosidade, foi utilizado um viscosímetro capilar de Cannon-Fenske Schott 51313 ( $\varnothing=0,77 \mathrm{~mm}$ ), imerso em um banho termostatizado a aproximadamente $25^{\circ} \mathrm{C}$.

O grau de polimerização $(G P)$ da celulose foi obtido pela Equação 2:

Tabela 1. Nomenclatura dos produtos celulósicos obtidos

\begin{tabular}{|c|c|c|c|}
\hline \multicolumn{2}{|c|}{ Celulose } & \multicolumn{2}{|c|}{ Acetato de celulose } \\
\hline Descrição & Nomenclatura & Descrição & Nomenclatura \\
\hline Celulose do bagaço de cana & $\mathrm{CBC}$ & Acetato de celulose do bagaço de cana & $\mathrm{ACBC}$ \\
\hline Celulose da bucha vegetal & $\mathrm{CBU}$ & Acetato de celulose da bucha vegetal & ACBU \\
\hline
\end{tabular}




$$
G P=\frac{M_{v}}{162 \mathrm{~g} \mathrm{~mol}^{-1}}
$$

sendo $162 \mathrm{~g} \mathrm{~mol}^{-1} \mathrm{o}$ valor da massa molar de uma unidade de anidro glucose.

\section{Determinação do grau de substituição (GS) dos acetatos de celulose}

Inicialmente, uma quantidade de $0,1 \mathrm{~g}$ da amostra de $\mathrm{AC}$ foi transferida para um Erlenmeyer e então $5 \mathrm{~mL}$ de uma solução padrão de $\mathrm{NaOH} 0,25 \mathrm{~mol} \mathrm{~L}^{-1}$ e $5 \mathrm{~mL}$ de etanol foram adicionados. Após $24 \mathrm{~h}, 10 \mathrm{~mL}$ de uma solução padrão de $\mathrm{HCl} 0,25 \mathrm{~mol} \mathrm{~L}^{-1}$ foram adicionados e, após $30 \mathrm{~min}$, o excesso do ácido foi titulado com uma solução padrão de $\mathrm{NaOH} 0,25 \mathrm{~mol} \mathrm{~L}^{-1}$, usando fenolftaleína como indicador. A partir dos volumes das soluções de $\mathrm{NaOH}$ e $\mathrm{HCl}$ usados em cada etapa da titulação, o valor de GS foi determinado de acordo com Puleo e colaboradores ${ }^{21}$ que se baseia na determinação da porcentagem de grupos acetila que foram substituídos na cadeia celulósica, conforme Equação 3. Cada experimento para determinar GS foi realizado em triplicata.

$$
\% G A=\frac{\left[\left(V b_{i}+V b_{t}\right) \mu_{b}-\left(V a \mu_{a}\right)\right] M \times 100}{m_{a c}}
$$

Nessa expressão: \%GA = porcentagem de grupos acetila substituídos, $\mathrm{Vb}_{i}=$ volume de $\mathrm{NaOH}$ adicionado, $\mathrm{Vb}_{t}=$ volume de $\mathrm{NaOH}$ obtido na titulação, $\mu_{b}=$ molaridade do $\mathrm{NaOH}, \mathrm{Va}=$ volume de $\mathrm{HCl}$ adicionado, $\mu_{a}=$ molaridade do $\mathrm{HCl}, \mathrm{M}=$ massa molar dos grupos acetila, $\mathrm{m}_{\mathrm{ac}}=$ massa do acetato utilizada.

Determinação da Massa Molar Viscosimétrica $\left(M_{v}\right)$ e do Grau de Polimerização $(G P)$ para o acetato de celulose

Para as amostras de acetato de celulose foi utilizado um sistema diclorometano/etanol (8/2 v/v) na concentração de $2,0 \mathrm{~g} \mathrm{~L}^{-1} \mathrm{de}$ acetato. ${ }^{32}$

Os valores de viscosidade foram obtidos de maneira idêntica à utilizada para as amostras de celulose. A massa molar viscosimétrica $\left(M_{v}\right)$ do acetato de celulose foi obtida pela Equação 4:

$$
[\eta]=K\left(M_{v}\right)^{\alpha} \text {, }
$$

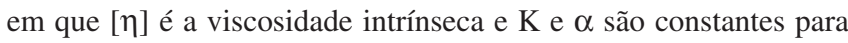
um par polímero-solvente em particular a uma dada temperatura. As constantes do sistema solvente utilizado são $\mathrm{K}=13,9 \times 10^{-3} \mathrm{~mL} \mathrm{~g}^{-1}$ e $\alpha=0,834 .{ }^{32}$

O grau de polimerização (GP) do acetato de celulose foi obtido pela Equação 5:

$$
G P=\frac{M_{v}}{M M_{A C}}
$$

sendo $M M_{A C}$ o valor da massa molar de um monômero de acetato de celulose considerando o grau de substituição do material acetilado.

\section{Análise por Espectroscopia de Ressonância Magnética Nuclear} ( $R M N)$ de ${ }^{13} C$ no estado sólido

Os experimentos de RMN no estado sólido com polarização cruzada (CP), desacoplamento heteronuclear e rotação em torno do ângulo mágico (MAS) foram realizados em um espectrômetro VarianAgilent $400 \mathrm{MHz}$, operando na frequência de 100,52 MHz para ${ }^{13} \mathrm{C}$ (campo magnético de 9,4 T). As amostras foram empacotadas em rotores de zircônia com $4 \mathrm{~mm}$ de diâmetro e colocadas no interior da sonda de radiofrequência à frequência de rotação de $10 \mathrm{kHz}$, com número de transientes de 750. Na sequência de pulsos utilizada nos experimentos de ${ }^{1} \mathrm{H}-{ }^{13} \mathrm{C} \mathrm{CP}$, a duração do pulso de $\pi / 2$ foi de $3,6 \mu \mathrm{s}$, o tempo de contato foi de $1 \mathrm{~ms}$, o tempo de repetição foi de $5 \mathrm{~s}$, o tempo de aquisição foi de $20,48 \mathrm{~ms}$ (durante o qual foi utilizado desacoplamento heteronuclear de núcleos ${ }^{1} \mathrm{H}$ ) e a janela espectral foi de $50 \mathrm{kHz}$. Os espectros foram obtidos por transformada de Fourier dos sinais no domínio do tempo, com uso de alargamento exponencial de $30 \mathrm{~Hz}$ e preenchimento com zeros até 4096 pontos.

\section{Análise Térmica}

As medidas de termogravimetria (TG) foram realizadas em um equipamento TGA-50 da Shimadzu a uma taxa de aquecimento de 10 ${ }^{\circ} \mathrm{C} \min ^{-1}$, da temperatura ambiente até $700{ }^{\circ} \mathrm{C}$, sob atmosfera de argô-

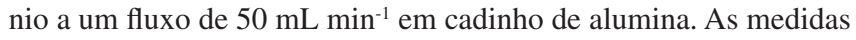
de calorimetria exploratória diferencial (DSC) foram realizadas em um equipamento DSC-50 da Shimadzu a uma taxa de aquecimento de $5^{\circ} \mathrm{C} \mathrm{min}{ }^{-1}$, da temperatura ambiente até $310^{\circ} \mathrm{C}$, sob atmosfera de

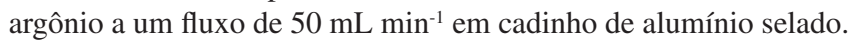

\section{Análise por Espectroscopia no Infravermelho por Transformada de Fourier (FTIR)}

Os experimentos de FTIR foram registrados utilizando-se a técnica de refletância total atenuada (ATR). Foi utilizado o espectrômetro da Agilent Technologies, modelo Cary 630 FTIR, registrando-se 32 scans na região de 4000 a $650 \mathrm{~cm}^{-1}{\mathrm{com} 4 \mathrm{~cm}^{-1} \mathrm{de}}$ resolução.

\section{Análise por Difração de Raios-X (DRX)}

Os experimentos de DRX foram realizados com as amostras de celulose e acetato obtidas em um difratômetro XRD-6000 Shimadzu com radiação $\mathrm{Cu}-\mathrm{K} \alpha(\lambda=0,15418 \mathrm{~nm})$, com ângulo de difração $2 \theta$ variando de 4 a $80^{\circ}$, passo de $0,02^{\circ}$ e velocidade de varredura de $2^{\circ} \mathrm{min}^{-1}$, utilizando-se porta-amostra de alumínio. A partir das medidas de DRX, foi determinado o índice de cristalinidade (Ic) para as amostras de celulose. Para isso, foi utilizado o método que se baseia na razão entre as amplitudes do pico de difração em $2 \theta \approx 23^{\circ}$ (denominada $I_{\text {máx }}$ ) e do mínimo localizado em torno de $2 \theta \approx 18^{\circ}$ (denominada $I_{\text {min }}$ ), de acordo com a Equação 6: ${ }^{33}$

$$
I c=\left[1-\left(\frac{I_{\min }}{I_{\text {máx }}}\right)\right] \times 100 \%
$$

\section{RESULTADOS E DISCUSSÃO}

\section{Rendimento do processo de extração da celulose}

Na Tabela 2 é apresentada a caracterização química dos precursores utilizados neste trabalho. O rendimento do processo de obtenção de celulose a partir da amostra CBC foi de $24,3 \%$. Se comparado com o teor de celulose no precursor BC (Tabela 2), o rendimento da extração de celulose foi de $73 \%$. Para a amostra CBU, o rendimento para o processo foi de $28 \%$, enquanto que o rendimento da extração de celulose foi de $56 \%$. Esses valores estão abaixo dos 94,6\% reportados por Suriyachai e colaboradores, ${ }^{34}$ que fracionaram o bagaço de cana usando o processo organossolve e ácido fórmico como catalisador. No entanto, estão próximos dos $60 \%$ relatados por Candido e Gonçalves, ${ }^{30}$ os quais usaram um pré-tratamento alcalino para extrair a celulose a partir do bagaço de cana. Provavelmente, esses baixos rendimentos do processo (24,3 e $28 \%$ para as amostras CBC e CBU, respectivamente) estejam relacionados com a razão massa do precursor lignocelulósico / volume dos solventes de extração. Esses valores sugerem que, para trabalhos futuros, a otimização do processo de extração deve ser individualizada para cada um dos precursores. 
Tabela 2. Caracterização química dos precursores lignocelulósicos. Os teores são fornecidos em porcentagem em massa, em base seca

\begin{tabular}{lcc}
\hline & Bagaço de cana in natura & Bucha vegetal in natura \\
\hline Cinzas (\%) & $4,51 \pm 0,06$ & $0,08 \pm 0,02$ \\
Extrativos (\%) & $2,6 \pm 0,4$ & $4,8 \pm 0,6$ \\
Lignina (\%) & $21,0 \pm 0,1$ & $15,0 \pm 0,2$ \\
Celulose (\%) & $37 \pm 1$ & $52 \pm 2$ \\
Hemicelulose (\%) & $34 \pm 1$ & $34 \pm 2$ \\
\hline
\end{tabular}

\section{Caracterização da celulose obtida para os precursores BC e BU}

Os valores de GP e $\mathrm{M}_{\mathrm{v}}$ foram determinados de acordo com o procedimento descrito na parte experimental. Os valores de GP para a celulose podem variar de acordo com o tratamento utilizado na obtenção do material. No caso da polpa de madeira, por exemplo, o valor típico de GP fica entre 300 e 1700, enquanto que para a celulose obtida a partir de outros precursores vegetais, como o algodão, esse valor encontra-se entre 800 e 10000. ${ }^{15}$ Esses valores são correlacionáveis com os valores de $\mathrm{M}_{\mathrm{v}}$ pela Equação 1. Como esperado, o GP das amostras de celulose deste estudo apresentaram valores distintos porque as biomassas precursoras são diferentes. $\mathrm{O}$ GP da amostra CBU foi de $1307 \pm 2$, enquanto que para a amostra $\mathrm{CBC}$ foi de $472 \pm 2$. O valor de GP para a amostra $\mathrm{CBC}$, quando comparado com a amostra CBU, é relativamente baixo. Contudo ele é ainda superior ao GP de amostras comerciais de celulose, como SigmaCell 20 que possui GP de $250 .^{35}$

A eficiência do processo de extração foi avaliada por RMN de ${ }^{13} \mathrm{C}$ no estado sólido, uma vez que essa técnica permite assinalar os seis carbonos presentes na estrutura da celulose, ${ }^{33,36}$ os carbonos atribuídos aos grupos aromáticos e ao grupo metoxila característicos da estrutura da lignina ${ }^{37}$ e os carbonos atribuídos aos grupos acetatos indicativos da presença de hemicelulose. ${ }^{38}$ A Figura 1 apresenta esses espectros. A obtenção de celulose com razoável pureza é evidenciada pela não observância de sinais associados à lignina nos deslocamentos químicos entre 110 e 150 ppm, (atribuídos aos grupos aromáticos) e próximo a 56 ppm (atribuído ao grupo metoxila), os quais são observados com grande intensidade nos espectros RMN de ${ }^{13} \mathrm{C}$ obtidos para os precursores lignocelulósicos. Também estão ausentes nos espectros das celuloses extraídas os sinais típicos de grupos acetato e metila característicos dos espectros de RMN de ${ }^{13} \mathrm{C}$ da hemicelulose (21,5 ppm e $174 \mathrm{ppm}$, respectivamente). Os espectros registrados para as amostras obtidas após as extrações (CBC e CBU) são típicos de materiais celulósicos com boa pureza e elevada cristalinidade, revelada pela existência de sinais relativamente estreitos e com acentuado desdobramento dos sinais correspondentes aos carbonos $\mathrm{C} 4$ e C6. ${ }^{33,36} \mathrm{Em}$ espectros de RMN de ${ }^{13} \mathrm{C}$ obtidos com o método CP/MAS em materiais lignocelulósicos, a presença de lignina é facilmente identificada pela observação dos sinais devidos aos grupos aromáticos e ao grupo metoxila. ${ }^{10,39} \mathrm{~A}$ não observância desses sinais indica que o teor de lignina residual nos espectros obtidos para os produtos CBC e CBU é reduzido; de fato, uma análise química desses produtos (conduzida de forma similar à utilizada para os precursores $)^{31}$ revelou teores de lignina na faixa 1-2\%, o que corresponde a uma severa redução em comparação com os teores correspondentes aos precursores lignocelulósicos (iguais a 18,9 e 13,4\% para os precursores BC e BU, respectivamente, como apresentado na Tabela 2).

\section{Caracterização dos acetatos de celulose obtidos para as amostras CBC e CBU}

Com base em discussões prévias já estabelecidas na literatura, ${ }^{40}$

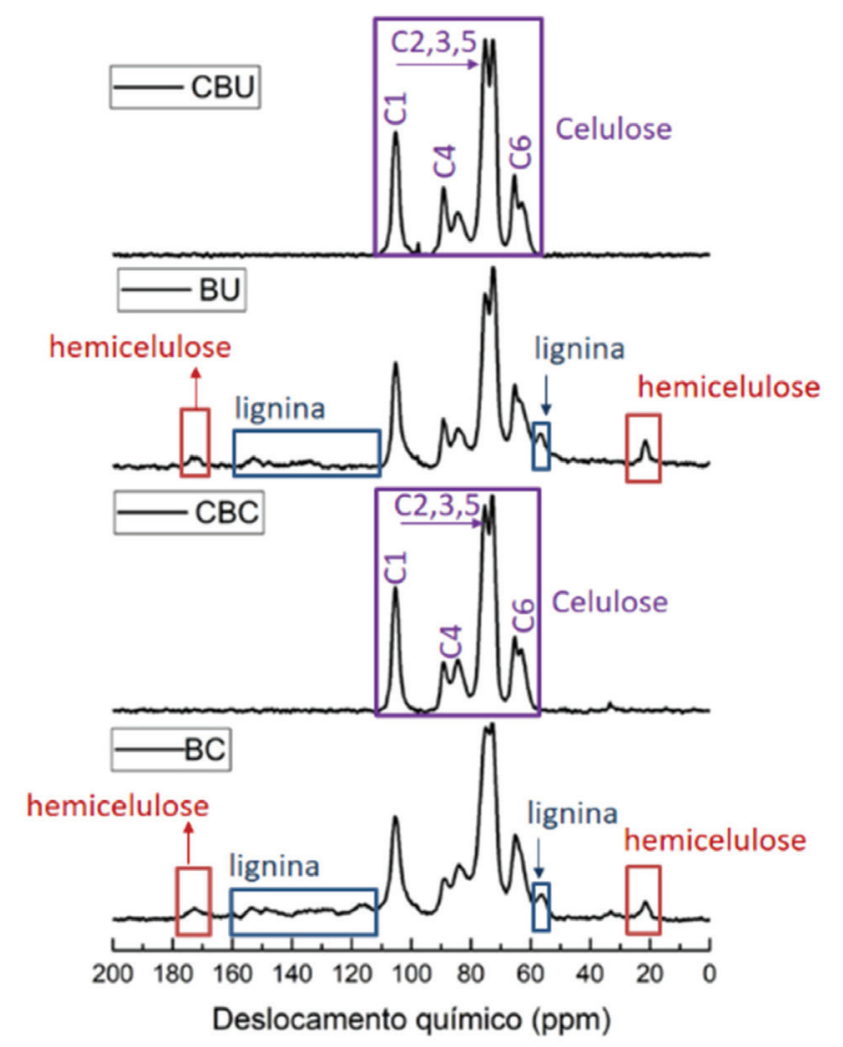

Figura 1. Espectros de $R M N{ }^{13} \mathrm{C}$ no estado sólido (CP/MAS) comparando os sinais dos precursores $B U$ e BC com sinais das celuloses obtidas após o processo de extração organossolve. Os símbolos C1 a C6 indicam os sinais associados aos vários tipos de carbono presentes nas unidades básicas de celulose e hemicelulose

os acetatos de celulose podem ser classificados como mono, di e triacetato de celulose, sendo os que possuem mais de $92 \%$ dos grupos hidroxila substituídos (isto é, com um GS $>2,76$ ), classificados como triacetato de celulose..$^{40}$ Neste trabalho, considerando as incertezas dos valores médios obtidos, foram produzidos dois triacetatos, com $\mathrm{GS}=2,64 \pm 0,12$ e 2,69 $\pm 0,08$ para as amostras de ACBC e ACBU, respectivamente. $\mathrm{O}$ valor de GS da amostra de acetato obtida a partir do bagaço de cana $(2,64 \pm 0,12)$ é praticamente igual aos valores de GS dos acetatos de celulose provenientes de precursores similares citados na literatura, tais como bagaço de cana $(2,52)^{14}$ e palha da cana de açúcar $(2,72 \pm 0,19) .^{30}$

Além disso, os acetatos de celulose foram caracterizados em relação ao seu grau de polimerização e, para isso, foram consideradas as massas molares do monômero de acetato de celulose em função dos valores de GS $\left(\mathrm{MM}_{\mathrm{AC}}=265 \pm 12 \mathrm{~g} \mathrm{~mol}^{-1}\right.$ para ACBC e $\mathrm{MM}_{\mathrm{AC}}=267 \pm 8 \mathrm{~g} \mathrm{~mol}^{-1}$ para ACBU). Acetatos de celulose tipicamente apresentam valores de GP na faixa de $150-300,{ }^{15,41}$ sendo valores inferiores aos graus de polimerização típicos para as celuloses precursoras, o que sugere uma degradação parcial das cadeias poliméricas durante o processo de acetilação. Os valores obtidos foram de $169 \pm$ 8 para a amostra ACBC (o que corresponde $\mathrm{M}_{\mathrm{v}}=45 \times 10^{3} \mathrm{~g} \mathrm{~mol}^{-1}$ ) e $337 \pm 10$ para a amostra ACBU (o que corresponde a $\mathrm{M}_{\mathrm{v}}=90 \times 10^{3}$ $\mathrm{g} \mathrm{mol}^{-1}$ ), seguindo a mesma tendência dos valores de GP para suas respectivas celuloses precursoras.

Finalmente, os rendimentos das reações de acetilação homogênea realizadas neste trabalho puderam ser determinados em $76 \%$ para a amostra ACBC e $83 \%$ para a amostra ACBU. Esses valores são comparáveis ao rendimento para a acetilação de amostras de celulose comercial e para amostras de celulose provenientes de diferentes tipos de biomassa. ${ }^{9,28,42,43}$ 
Na Figura 2 são apresentados os espectros de FTIR para os produtos celulósicos preparados. Os espectros para as amostras $\mathrm{CBC}$ e CBU apresentaram bandas características de celulose ${ }^{44}$ sugerindo eficiente processo de extração. Isso é evidenciado pela não observância do padrão de bandas entre 1700 e $1420 \mathrm{~cm}^{-1}$ característico da lignina, faixa que contém as bandas de 1600 e $1455 \mathrm{~cm}^{-1}$ atribuídas ao estiramento $\mathrm{C}=\mathrm{C}$ de anel aromático, e a banda em $1424 \mathrm{~cm}^{-1}$, atribuída ao estiramento $\mathrm{O}-\mathrm{CH}_{3}$ de metoxila, que são intensas no espectro de FTIR da lignina. ${ }^{44-46} \mathrm{As}$ principais diferenças observadas entre os espectros dos acetatos de celulose e das celuloses foram o aparecimento de uma banda de forte intensidade em $1737 \mathrm{~cm}^{-1}$, característica do estiramento $\mathrm{C}=\mathrm{O}$ presentes no acetato de celulose e da banda em $1213 \mathrm{~cm}^{-1}$, atribuída ao estiramento C-O da acetila. Observa-se também o desaparecimento da banda em $3310 \mathrm{~cm}^{-1}$ atribuída ao estiramento das ligações de hidrogênio intermolecular entre as hidroxilas celulósicas, o que corrobora os resultados obtidos na determinação de GS, indicando que as hidroxilas foram substituídas pelos grupos acetila. ${ }^{46}$

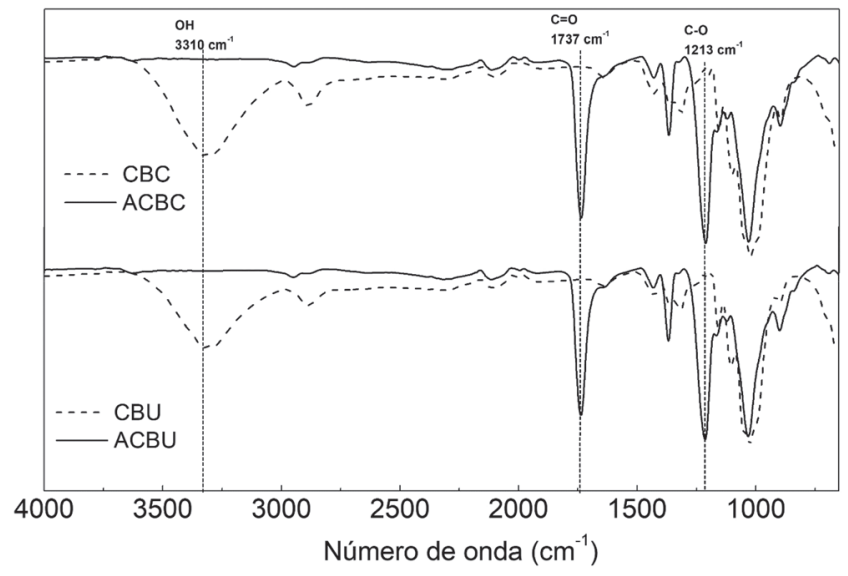

Figura 2. Espectros de FTIR das amostras de celulose e acetatos de celulose

O índice de cristalinidade (Ic) é um parâmetro bastante utilizado para interpretar modificações estruturais na celulose submetida a tratamentos físico-químicos. ${ }^{47}$ Os valores Ic dos materiais celulósicos são tipicamente determinado por DRX..$^{33}$ Outros métodos também apropriados (e em muitos casos preferíveis) são as espectroscopias de RMN no estado sólido, ${ }^{48} \operatorname{Raman}^{47}$ e FTIR. ${ }^{47}$ Além disso, estudos recentes indicam que os valores de Ic podem ser influenciados pela quantidade de água absorvida na celulose e pela organização espacial das cadeias celulósicas interagindo via ligações de hidrogênio após o processo de extração. ${ }^{47}$

Neste trabalho foi utilizado o método das alturas dos picos de difração proposto por Park e colaboradores ${ }^{33}$ para avaliar os índices de cristalinidade das amostras de celulose. Os valores de Ic calculados para as amostras CBC e CBU foram de 73 e 78,4\%, respectivamente. Esses valores são comparáveis com os valores reportados na literatura para materiais similares, tipicamente variando entre 78 e $95 \% .{ }^{33} \mathrm{O}$ menor valor de Ic para a amostra CBC, se comparada com a CBU, está de acordo com o menor valor de GP obtido para a mesma amostra, indicando que, após a extração, a celulose obtida a partir do precursor BU possui uma mais elevada cristalinidade e um maior grau de polimerização do que o produto obtido do precursor BC..$^{35,47}$ Esse resultado também é consistente com a observação dos sinais devidos aos carbonos $\mathrm{C} 4$ e C6 em regiões estruturalmente ordenadas e desordenadas de celulose nos espectros de $\mathrm{RMN}$ de ${ }^{13} \mathrm{C}$ mostrados na Figura $1 .^{10,36}$ De fato, observa-se que os sinais em torno de $66 \mathrm{ppm}$ (carbono C6 em regiões ordenadas) e 89 ppm (carbono C4 em regiões ordenadas) são relativamente mais intensos no espectro obtido para a amostra CBU do que na amostra CBC, em comparação com os sinais detectados em torno de 63 ppm (carbono $\mathrm{C} 4$ em regiões desordenadas) e 84 ppm (carbono $\mathrm{C} 4$ em regiões desordenadas).

A Figura 3 apresenta um comparativo entre os difratogramas de raios-X da celulose antes e após a reação de acetilação. A presença de picos muito mais alargados nos difratogramas obtidos para os acetatos sugere que a reação de acetilação promoveu mudanças na cristalinidade do material (ordenamento das cadeias celulósicas interagindo via ligações de hidrogênio), indicando que a estrutura dos acetatos é consideravelmente menos organizada do que nas celuloses precursoras. Os picos alargados que ocorrem em $2 \theta=8,5^{\circ} ; 17,7^{\circ} \mathrm{e}$ $21,8^{\circ}$ são característicos do acetato de celulose. ${ }^{11} \mathrm{O}$ pico em $8,5^{\circ}$ é tido como o principal pico de derivados acetilados semicristalinos de celulose. ${ }^{20}$

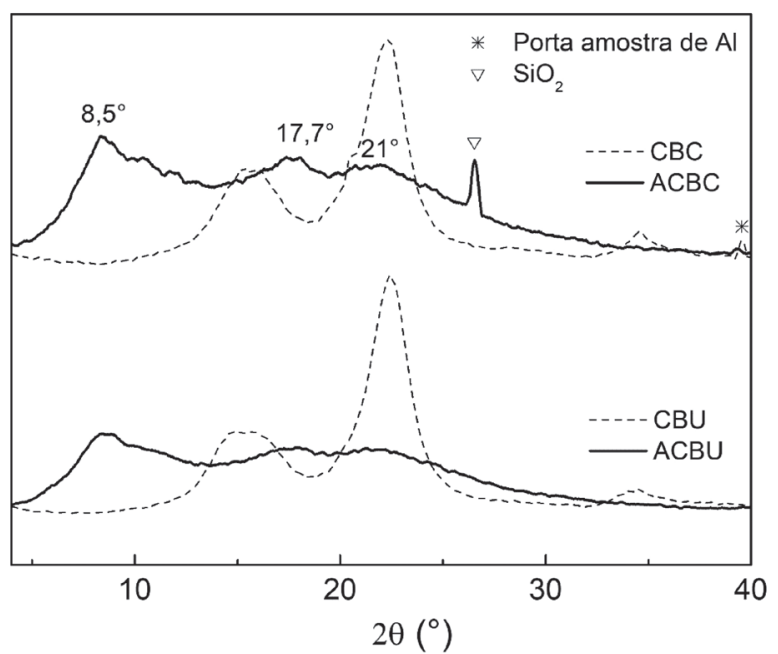

Figura 3. Difratogramas de raios- $X$ registrados para as amostras secas de celulose ( $C B U$ e $C B C$ ) e dos acetatos de celulose correspondentes ( $A C B U$ e $A C B C$, respectivamente)

Na Figura 4 são apresentados os espectros de $\mathrm{RMN}$ de ${ }^{13} \mathrm{C}$ no estado sólido dos acetatos obtidos, assim como das celuloses precursoras. As principais diferenças observadas nos espectros correspondentes aos acetatos de celulose com relação aos espectros das celuloses dizem respeito aos sinais em 170,6 ppm (atribuído a grupos carbonila) e em

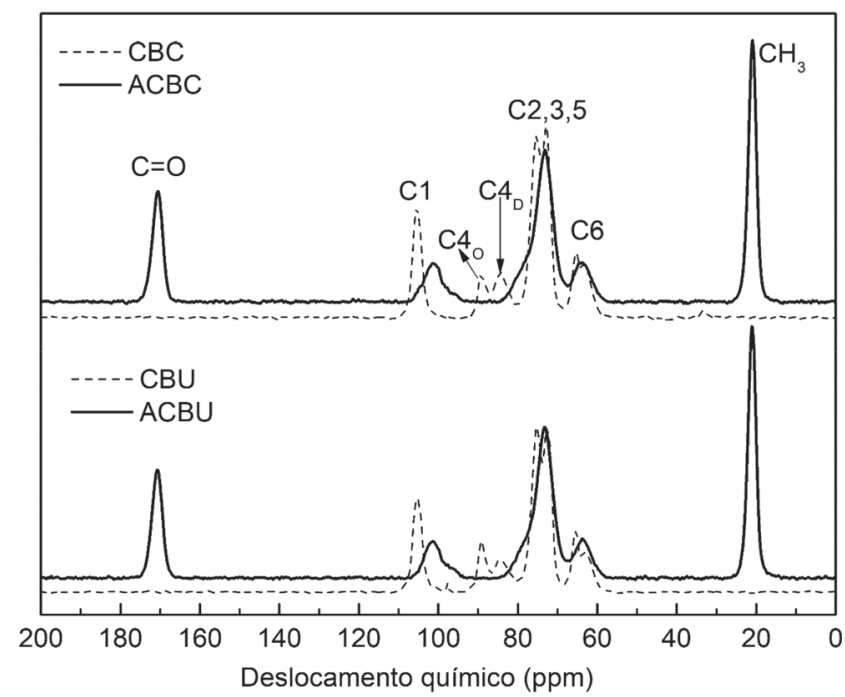

Figura 4. Espectros de RMN de ${ }^{13} \mathrm{C}$ no estado sólido (CP/MAS) obtidos para os acetatos de celulose ( $A C B U$ e $A C B C$ ) em comparação com as respectivas celuloses ( $C B U$ e $C B C)$ 
21,0 ppm (atribuído a grupos metila), evidenciando a presença dos grupos acetila nas amostras de acetato de celulose. ${ }^{10}$

Foram observadas modificações nos sinais correspondentes aos deslocamentos químicos da celulose após a acetilação. O sinal atribuído ao carbono C1 apresenta um deslocamento de 105,2 ppm para 101,4 ppm, além de uma diminuição na intensidade e aumento da largura. No caso dos espectros de celulose, a região associada aos carbonos C2, C3 e C5 se apresenta com dois sinais (75,2 ppm e 72,6 ppm), enquanto que, nos espectros dos acetatos de celulose, essa região apresenta-se como um singleto em 73,2 ppm, abrangendo também o sinal atribuído ao carbono $\mathrm{C} 4$. Nos espectros da celulose, por outro lado, o carbono C4 contribui com os sinais em 89,1 ppm, atribuído ao carbono $\mathrm{C} 4$ da celulose em regiões de elevada ordem estrutural $\left(\mathrm{C}_{\mathrm{O}}\right)$ e 83,3 ppm, atribuído ao carbono $\mathrm{C} 4$ da celulose em regiões menos ordenadas $\left(\mathrm{C}_{\mathrm{D}}\right)$. Ainda, o sinal atribuído ao carbono C6 (65,3 ppm) apresenta uma diminuição na intensidade no caso dos espectros obtidos para os acetatos..$^{20,49}$ Essas alterações já foram previamente reportadas em outros trabalhos da literatura, sendo interpretadas como indicadoras da ocorrência de modificações químicas e estruturais no material associadas ao processo de acetilação. ${ }^{50-52}$

Nas curvas de TG e DSC obtidas para as amostras de acetatos de celulose (Figura 5), foi possível observar uma perda inicial de massa na ordem de 1 a $2 \%$, referente à saída de água adsorvida ao material, por meio de um processo endotérmico que ocorre até em torno de $100{ }^{\circ} \mathrm{C}$. Por volta de $193{ }^{\circ} \mathrm{C}$, ocorre um evento exotérmico correspondente à cristalização do material, que não é observada para as amostras de celulose (Figura 1S), ${ }^{53}$ temperatura denominada Tc. Em seguida, ocorre um evento endotérmico por volta de $282{ }^{\circ} \mathrm{C}$, correspondente à fusão do material. Na curva de TG, é possível observar que a partir de aproximadamente $310{ }^{\circ} \mathrm{C}$ o material sofre uma brusca perda de massa de $80 \%$. À medida que a temperatura é ainda mais elevada, o material sofre perdas de massa mais suaves até um resíduo de aproximadamente $2 \%$ à temperatura final de $700{ }^{\circ} \mathrm{C}$. Esses últimos eventos são associados à degradação do material.
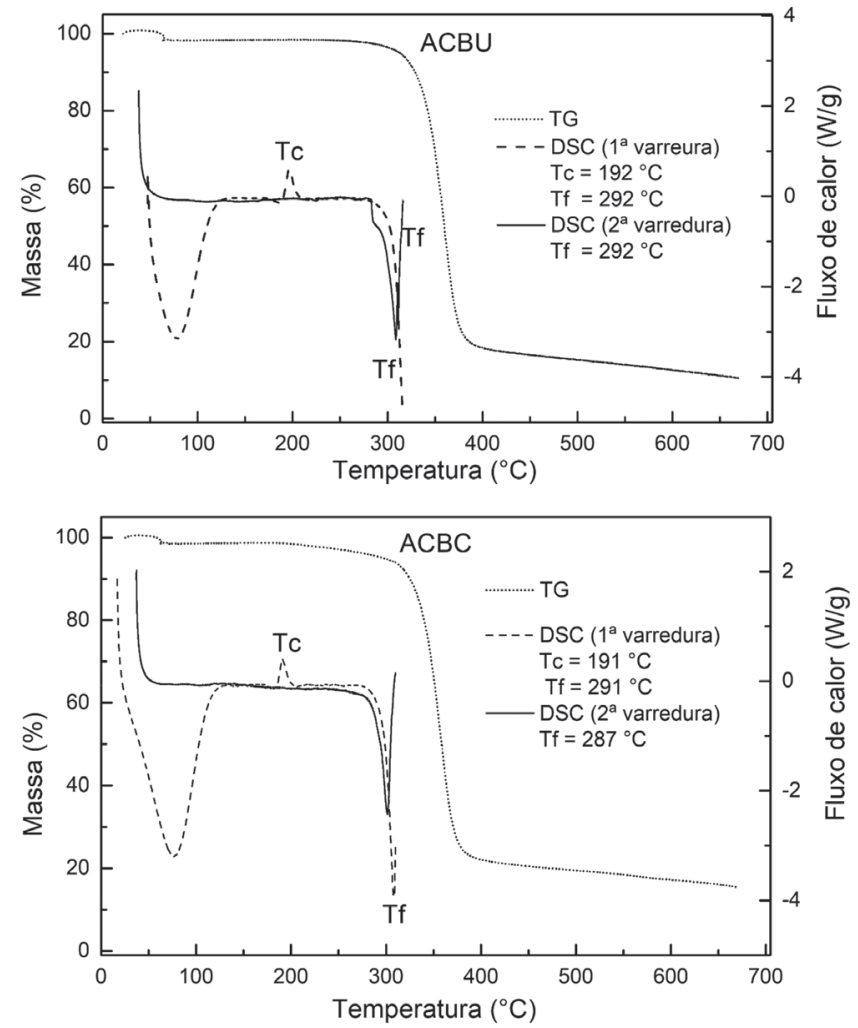

Figura 5. Curvas de TG e DSC para as amostras de acetato $A C B U$ e ACBC
As medidas de DSC não foram levadas a esse extremo a fim de se avaliar a reversibilidade dos processos de cristalização e fusão do material, fazendo-se uma segunda varredura. É possível notar que o material foi completamente cristalizado durante o primeiro tratamento térmico, não sendo observado esse evento na segunda varredura, e que o processo de fusão é reversível, ocorrendo a uma temperatura bem próxima daquela observada no primeiro tratamento térmico. ${ }^{14}$

Os resultados de caracterização térmica para os dois acetatos obtidos (ACBU e ACBC) apresentaram perfis semelhantes e mostram a obtenção de materiais com boa estabilidade térmica, corroborando os resultados reportados na literatura para acetatos com alto grau de substituição. ${ }^{13,14}$

Os resultados de caracterizações por FTIR, ${ }^{13} \mathrm{C}$-RMN e análise térmica dos materiais obtidos revelaram que a extração e a acetilação da celulose foram bem-sucedidas para o caso dos dois precursores investigados. As amostras de acetato de celulose obtidas apresentaram aparência similar (como ilustrado nas imagens exibidas no Graphical Abstract), sendo observada uma coloração ligeiramente distinta por conta do grau de aglomeração das partículas presentes nos produtos obtidos a partir dos precursores BC e BU. Os maiores valores de GP e Ic para a celulose e GP e rendimento da acetilação para o acetato de celulose produzidos a partir da bucha vegetal sugerem que esse material constitui um precursor de baixo custo e potencialmente útil para a obtenção de derivados de celulose.

\section{CONCLUSÃO}

Neste trabalho, a metodologia organossolve mostrou ser uma boa alternativa para a extração de celulose com razoável pureza e elevada cristalinidade (Ic acima de 70\%). Evidenciou-se que os produtos celulósicos obtidos a partir da bucha vegetal apresentaram cristalinidade superior, quando comparados aos produtos obtidos a partir do bagaço de cana. As celuloses extraídas foram acetiladas com eficiência, conforme demonstrado por análises de FTIR, $\mathrm{RMN}$ de ${ }^{13} \mathrm{C}$ no estado sólido. Além disso, a cristalinidade e as propriedades térmicas foram avaliadas para as amostras de acetato de celulose produzidas. Finalmente, os acetatos produzidos foram caracterizados em relação ao rendimento da reação de 76 e $83 \%$; $\mathrm{GS}=2,64 \pm 0,12$ e 2,69 $\pm 0,08$; massa molar viscosimétrica de 45 $\times 10^{3} \mathrm{~g} \mathrm{~mol}^{-1} \mathrm{e} 90 \times 10^{3} \mathrm{~g} \mathrm{~mol}^{-1}$, respectivamente. Esses resultados sugerem que a metodologia usada neste trabalho foi eficiente para obtenção de acetatos de celulose com elevado grau de substituição a partir do bagaço de cana e da bucha vegetal, sendo apropriada, portanto, para a produção de materiais avançados de elevado interesse tecnológico.

\section{MATERIAL SUPLEMENTAR}

Os resultados obtidos nas análises térmicas (TG e DSC) para as amostras de celulose obtidas neste trabalho estão disponíveis em http://quimicanova.sbq.org.br, na forma de arquivo PDF, com acesso livre.

\section{AGRADECIMENTOS}

O presente trabalho foi realizado com apoio da Coordenação de Aperfeiçoamento de Pessoal de Nível Superior - Brasil (CAPES) Código de Financiamento 001. Os autores gostariam de agradecer ao LabPetro (UFES, Brasil) pela realização das análises de FTIR (termo de Cooperação no. 0050.0022844.06.4). Agradecemos também à Universidade Federal do Espírito Santo (UFES) e ao Centro Universitário Norte do Espírito Santo (CEUNES); às agências de apoio e fomento à pesquisa: Conselho Nacional de Desenvolvimento 
Científico e Tecnológico (CNPq), Fundação de Amparo à Pesquisa e Inovação do Espírito Santo (FAPES).

\section{REFERÊNCIAS}

1. Ferreira, V. F.; De Carvalho Da Silva, F.; Ferreira, P. G.; Quim. Nova 2013, 36, 1514.

2. Da Rocha, G. O.; De Andrade, J. B.; Guarieiro, A. L. N.; Guarieiro, L. L. N.; Ramos, L. P.; Quim. Nova 2013, 36, 1540.

3. Galembeck, F.; Quim. Nova 2013, 36, 1600.

4. Santos, F. A.; de Queiróz, J. H.; Colodette, J. L.; Fernandes, S. A.; Guimarães, V. M.; Rezende, S. T.; Quim. Nova 2012, 35, 1004.

5. Marengo, V. A.; Vercelheze, A. E. S.; Mali, S.; Quim. Nova 2013, 36, 680.

6. Ioannidou, O.; Zabaniotou, A.; Renewable Sustainable Energy Rev. 2007, 11, 1966.

7. Hahn-Hägerdal, B.; Galbe, M.; Gorwa-Grauslund, M. F.; Lidén, G.; Zacchi, G.; Curr. Trends Biotechnol. 2006, 24, 549.

8. Abraham, E.; Weber, D. E.; Sharon, S.; Lapidot, S.; Shoseyov, O.; ACS Appl. Mater. Interfaces. 2017, 9, 2010.

9. Ragab, S.; El Nemr, A.; J. Macromol. Sci., Part A: Pure Appl. Chem. 2018, 55, 124 .

10. Amaral, H. R.; Cipriano, D. F.; Santos, M. S.; Schettino Jr., M. A.; Ferreti, J. V. T.; Meirelles, C. S.; Pereira, V. S.; Cunha, A. G.; Emmerich, F. G.; Freitas, J. C. C.; Carbohydr. Polym. 2019, 210, 127.

11. Shi, Y.; Li, C.; He, D.; Shen, L.; Bao, N.; J. Mater. Sci. 2017, 52, 13296.

12. Gavilà, L.; Esposito, D.; Green Chem. 2017, 19, 2496.

13. Cao, L.; Luo, G.; Tsang, D. C. W.; Chen, H.; Zhang, S.; Chen, J.; J. Cleaner Prod. 2018, 176, 338.

14. Candido, R. G.; Godoy, G. G.; Gonçalves, A.; Carbohydr. Polym. 2017, 167, 280.

15. Credou, J.; Berthelot, T.; J. Mater. Chem. B 2014, 2, 4767.

16. https://www.researchandmarkets.com/research/g7b66s/global_cellulose, acessada em Fevereiro 2019

17. Chou, W. L.; Yu, D. G.; Yang, M. C.; Jou, C. H.; Sep. Purif. Technol. 2007, 57, 209.

18. Garg, A.; Gupta, M.; Bhargava, H. N.; Eur. J. Pharm. Biopharm. 2007, 67,725 .

19. Sossna, M.; Hollas, M.; Schaper, J.; Scheper, T.; J. Membr. Sci. 2007, 289,7

20. Barud, H. S.; Araújo Júnior, A. M.; Santos, D. B.; Assunção, R. M. N.; Meireles, C. S.; Cerqueira, D. A.; Rodrigues Filho, G.; Ribeiro, C. A.; Messaddeq, Y.; Ribeiro, S. J. L.; Thermochim. Acta. 2008, 471, 61.

21. Puleo, A. C.; Paul, D. R.; Kelley, S. S.; J. Membr. Sci. 1989, 47, 301.

22. de Melo Brites, M.; Ceróna, A. A.; Costa, S. M.; Oliveira, R. C.; Ferraz, H. G.; Catalani, L. H.; Costa, S. A.; Enzyme Microb. Technol. 2020, 132, 109384.

23. Ramphul, H.; Bhaw-Luximon, A.; Jhurry, D.; Carbohydr. Polym. 2017, $178,238$.

24. Chen, M. J.; Li, R. M.; Zhang, X. Q.; Feng, J.; Feng, J.; Liu, C. F.; Shi, Q. S.; ACS Sustainable Chem. Eng. 2017, 5, 360.
25. Das, A. M.; Ali, A. A.; Hazarika, M. P.; Carbohydr. Polym. 2014, 112, 342.

26. Rowell, R. M.; Wood Mater. Sci. Eng. 2006, 1, 29.

27. https://www.ibge.gov.br/estatisticas/economicas/agricultura-epecuaria/9201-levantamento-sistematico-da-producao-agricola. html?=\&t=downloads, acessado em Fevereiro 2020.

28. Candido, R. G.; Gonçalves, A. R.; Carbohydr. Polym. 2016, 152, 679.

29. Satyanarayana, K. G.; Guimarães, J. L.; Wypych, F.; Composites, Part A 2007, 38, 1694.

30. Guimarães, J. L.; Frollini, E., da Silva, C. G.; Wypych, F.; Satyanarayana, K. G.; Ind. Crops Prod. 2009, 30, 407.

31. Morais, J. P. S.; Rosa, M. F.; Marconcini, J. M.; Procedimentos para Análise Lignocelulósica, $1^{\mathrm{a}}$ ed., Embrapa Algodão: Campina Grande, 2010.

32. Knaus, S.; Bauer-Heim, B.; Carbohydr. Polym. 2003, 53, 383.

33. Park, S.; Baker, J. O.; Himmel, M. E.; Parilla, P. A.; Johnson, D. K.; Biotechnol. Biofuels 2010, 3, 10.

34. Suriyachai, N.; Champreda, V.; Kraikul, N.; Techanan, W.; Laosiripojana, N.; 3 Biotech 2018, 8, 221.

35. Mattonai, M.; Pawcenis, D.; del Seppia, S.; Łojewska, J.; Ribechini, E.; Bioresour. Technol. 2018, 270, 270.

36. Park, S.; Johnson, D. K.; Ishizawa, C. I.; Parilla, P. A.; Davis, M. F.; Cellulose 2009, 16, 641.

37. Wen, J. L.; Sun, S. L.; Xue, B. L.; Sun, R. C.; Materials 2013, 6, 359.

38. Foston, M.; Curr. Opin. Biotechnol. 2014, 27, 176

39. Lima, M. A.; Gomez, L. D.; Steele-King, C. G.; Simister, R.; Bernardinelli, O. D.; Carvalho, M. A.; Rezende, C. A.; Labate, C. A.; de Azevedo, E. R.; McQueen-Mason, S. J.; Polikarpov, I.; Biotechnol. Biofuels 2014, 7.

40. Gnanou, Y.; Fontanille, M.; Organic and Physical Chemistry of Polymers. $1^{\text {st }}$ ed., Wiley: New Jersey, 2008.

41. Höfte, H.; Voxeur, A.; Curr. Biol. 2017, 27, R865.

42. Hindi, S. S. Z.; Abohassan, R. A.; BioResources 2015, 10, 5030.

43. El Nemr, A.; Ragab, S.; El Sikaily, A.; Iran Polym. J. 2017, 26, 261.

44. Yang, H.; Yan, R.; Chen, H.; Lee, D. H.; Zheng, C.; Fuel 2007, 86, 1781.

45. Lopes, W. A.; Fascio, M.; Quim. Nova 2004, 27, 670.

46. Pavia, D. L.; Lampman, P.; Vyvyan, K.; Kriz, G. S.; Introduction to Spectroscopy, $4^{\text {th }}$ ed., Brooks/Cole: Belmont, 2009.

47. Agarwal, U. P.; Ralph, S. A.; Reiner, R. S.; Baez, C.; Carbohydr. Polym. 2018, 190, 262.

48. Brinkmann, A.; Chen, M.; Couillard, M.; Jakubek, Z. J.; Leng, T.; Johnston, L. J.; Langmuir 2016, 32, 6105.

49. Kono, H.; Numata, Y.; Nagai, N.; Erata, T.; Takai, M.; Carbohydr. Res. 1999, 322, 256

50. Yamamoto, H.; Horii, F.; Hirai, A.; Cellulose 2006, 13, 327.

51. Kono, H.; Erata, T.; Takai, M.; J. Am. Chem. Soc. 2002, 124, 7512.

52. Kono, H.; Hashimoto, H.; Shimizu, Y.; Carbohydr. Polym. 2015, 118, 91.

53. Calahorra, M. E.; Cortázar, M.; Eguiazábal, J. I.; Guzmán, G. M. T.; J. Appl. Polym. Sci. 1989, 37, 3305. 